\title{
Terrestrial exospheric hydrogen density distributions under solar minimum and solar maximum conditions observed by the TWINS stereo mission
}

\author{
J. H. Zoennchen, U. Nass, and H. J. Fahr \\ Argelander Institut für Astronomie, Astrophysics Department, University of Bonn, Auf dem Huegel 71, \\ 53121 Bonn, Germany
}

Correspondence to: J. H. Zoennchen (zoenn@ astro.uni-bonn.de)

Received: 13 November 2014 - Revised: 21 February 2015 - Accepted: 2 March 2015 - Published: 27 March 2015

\begin{abstract}
Circumterrestrial Lyman- $\alpha$ column brightness observations above 3 Earth radii $\left(R_{\mathrm{e}}\right)$ have been used to derive separate 3-D neutral hydrogen density models of the Earth's exosphere for solar minimum $(2008,2010)$ and near-solarmaximum (2012) conditions. The data used were measured by Lyman- $\alpha$ detectors (LAD1/2) onboard each of the TWINS satellites from very different orbital positions with respect to the exosphere. Exospheric $\mathrm{H}$ atoms resonantly scatter the near-line-center solar Lyman- $\alpha$ flux at $121.6 \mathrm{~nm}$. Assuming optically thin conditions above $3 R_{\mathrm{e}}$ along a line of sight (LOS), the scattered LOS-column intensity is proportional to the LOS H-column density. We found significant differences in the density distribution of the terrestrial exosphere under different solar conditions. Under solar maximum conditions we found higher $\mathrm{H}$ densities and a larger spatial extension compared to solar minimum. After a continuous, 2-month decrease in (27 day averaged) solar activity, significantly lower densities were found. Differences in shape and orientation of the exosphere under different solar conditions exist. Above $3 R_{\mathrm{e}}$, independent of solar activity, increased $\mathrm{H}$ densities appear on the Earth's nightside shifted towards dawn. With increasing distance (as measured at $8 R_{\mathrm{e}}$ ) this feature is shifted westward/duskward by between -4 and $-5^{\circ}$ with respect to midnight. Thus, at larger geocentric distance the exosphere seems to be aligned with the aberrated Earth-solar-wind line, defined by the solar wind velocity and the orbital velocity of the Earth. The results presented in this paper are valid for geocentric distances between 3 and $8 R_{\mathrm{e}}$.
\end{abstract}

Keywords. Atmospheric composition and structure (airglow and aurora; pressure density and temperature) - meteorology and atmospheric dynamics (thermospheric dynamics)

\section{Introduction}

Neutral hydrogen atoms are the main component of the terrestrial exosphere. They produce the geocoronal Lyman- $\alpha$ glow by resonant scattering of solar Lyman- $\alpha$ radiation at $121.6 \mathrm{~nm}$. In the optically thin regime above 3 Earth radii $\left(R_{\mathrm{e}}\right)$, the geocoronal Lyman- $\alpha$ column brightness is proportional to the corresponding neutral $\mathrm{H}$-column density along a line of sight (LOS). Observation of geocoronal Lyman- $\alpha$ column brightness and its conversion into exospheric H-density models have challenged researchers for decades. In the past, observations have been carried out with high-altitude rockets or satellites (e.g., Kupperian et al., 1959; Johnson, 1961; Rairden et al., 1986; Østgaard et al., 2003) and even by Apollo 16 from the Moon (Carruthers et al., 1976). Theoretical studies have been presented (e.g., Chamberlain, 1963; Thomas and Bohlin, 1972; Fahr and Shizgal, 1983; Bishop, 1991; Hodges Jr., 1994).

The Lyman- $\alpha$ detectors (LADs) onboard the two TWINS (Two Wide-angle Imaging Neutral-atom Spectrometers) satellites (McComas et al., 2009) have provided nearly continuous, circumterrestrial Lyman- $\alpha$ observations of the exosphere (from $\approx 7.2 R_{\mathrm{e}}$ apogee) since 2008 . These observations represent a unique database to determine the longperiod behavior of the exospheric 3-D density structure under different solar activities. 
Based on TWINS LAD data for the last solar minimum (2008, 2010), earlier studies (e.g., Bailey and Gruntman, 2011; Zoennchen et al., 2011, 2013) presented the first exospheric density models with angular dependence in the optically thin regime. In the present study we extend the analysis to a period near solar maximum (2012) and implement several new data corrections. Two typically different exospheres are fitted (separately for solar minimum and maximum) and are compared with each other. This revised analysis corrects the LAD data for geocoronal multiple-scattering effects (self-absorption and re-emission) using a multiplescattering Monte Carlo model based on "coherent line-center scattering" of photons in order to calculate the locally effective solar illumination. We will show that by taking these effects into account, the nightside observational coverage is improved, so that LOSs near the Earth's penumbra region no longer need to be excluded from the model fit, as was the case in many earlier studies. Also, new methods are introduced to recalibrate the TWINS LAD sensitivity based on observed stellar UV peaks and to mask UV-bright stars for exclusion from the data sets.

\section{Mission performance}

The TWINS 1 and 2 satellites have occupied high-elliptic Molniya orbits around the Earth (apogee $\approx 7.2 R_{\mathrm{e}}$ ) since the middle of 2008. Both satellites are in different orbits with an angular shift of their apogee positions of about $35^{\circ}$. In every orbit the LADs are activated for observation as soon as they are at geocentric distances above $4.5 R_{\mathrm{e}}$ (see details in Nass et al., 2006). When in the observing mode, the satellites positions are in the Northern Hemisphere with view directions always towards south. Mounted on a $180^{\circ}$ forward- and backward-rotating actuator, the two LADs per TWINS satellite observe the exosphere under a tilt angle of $40^{\circ}$ (field of view $4^{\circ}$ ) with respect to the Earth. A full circle (band of $4^{\circ}$ width) on the sky is observed after $\approx 90 \mathrm{~s}$. The time between two individual LAD observations is $0.67 \mathrm{~s}$.

LOSs with angles $\leq 90^{\circ}$ with respect to the Sun are contaminated with solar Lyman- $\alpha$ stray-light radiation and therefore have to be excluded. This significantly reduces the observational coverage of the Earth's dayside and the number of total usable Lyman- $\alpha$ data. Aging, thermal effects and other influences during the mission period continuously change the LAD sensitivity, which has been taken into account in the analysis.

\section{Approach}

To model the 3-D hydrogen density distribution of the terrestrial exosphere, geocoronal Lyman- $\alpha$ column brightness observations by TWINS 1 and 2 LADs within the optically thin regime above $3 R_{\mathrm{e}}$ have been used. These data were corrected for different effects: geocoronal Lyman- $\alpha$ self-absorption and re-emission from lower (optically thick) regions, contamination by UV-bright stars, interplanetary Lyman- $\alpha$ background, sensitivity changes in the LADs and an angle-dependent scattering phase function. These effects are discussed in detail in the following sections.

Through use of the function given by Emerich et al. (2005), the daily line-center solar Lyman- $\alpha$ flux was calculated from the total solar Lyman- $\alpha$ flux as measured by TIMED SEE and SORCE SOLSTICE instruments. From the daily line-center solar Lyman- $\alpha$ flux, the $g$ factor (resonant scattering rate) can be derived and used normalized to the daily heliocentric Earth distance as shown in Eq. (1).

Following Anderson and Hord Jr. (1977), a medium with an optical depth $\tau$ on the order of 0.1 or less can be defined as optically thin, since $90 \%$ of the photons are not influenced by multiple-scattering effects. The two presented H-density model fits for solar minimum and solar maximum have $\tau$ values of about 0.085 and 0.12 , respectively (radially integrated between 3 and $8 R_{\mathrm{e}}$ and angularly averaged). However, the tilted observational geometry of the TWINS LAD influences the integrated $\tau$-value of the LOS, in particular in regions with greater distances from the detectors. LOSs which pass the Earth above a certain distance $\left(\geq 4.6 R_{\mathrm{e}}\right.$ for the solar minimum model and $\geq 5.3 R_{\mathrm{e}}$ for the solar maximum model) have angularly averaged LOS-integrated $\tau$ values less than 0.15 (within $r \leq 8 R_{\mathrm{e}}$ ). LOSs with lower Earth impact parameters (closest distance of the LOS to the Earth center) at 3-4 $R_{\mathrm{e}}$ have $\tau$ values up to 0.15 in the LOS region between the detector and the Earth impact point. The majority of the LOS photons have their origins in the zone near the Earth impact point. In the more distant regions between the Earth impact point and the outer model boundary, those LOSs can have integrated $\tau$ values above 0.15 . However, since observations were taken from multiple and very different spacecraft positions, there is a large probability that a given geocoronal region is observed under different $\operatorname{LOS} \tau$ values. In those cases an average $\tau$-value is in effect in the model fits.

Under the assumption of single scattering for a particular solar Lyman- $\alpha$ radiation (manifested in the $g$ factor) the exospheric $\mathrm{H}$ density $N(S)$ along a LOS $S$ produces a Lyman- $\alpha$ scatter intensity $I$ in rayleigh $(\mathrm{R})(1 \mathrm{R}=$ $10^{6} /(4 \pi)$ photons $\left.\mathrm{cm}^{-2} \mathrm{~s}^{-1} \mathrm{sr}^{-1}\right)$ :

$I=\frac{g}{10^{6}} \int_{0}^{S_{\max }} N(S) \epsilon(S) I_{\mathrm{p}}[\alpha(S)] \mathrm{d} S$,

where $N(S)$ is the local $\mathrm{H}$ density, $\epsilon(S)$ the local correction term for geocoronal self-absorption/re-emission and $I_{\mathrm{p}}[\alpha(S)]$ the local intensity correction for the angular dependence of the scattering as presented in Eq. (4). We integrate along the LOS up to $25 R_{\mathrm{e}}$. Since we use the single-scattering approximation, the scattered photons are assumed to reach the detector without any participation in further scattering or loss processes. To improve this analysis, we introduce with 
$\epsilon(S)$ the quantitative treatment of the Lyman- $\alpha$ absorption and re-emission features from the lower, optically thick exospheric regions.

An important research aim is to investigate how exospheric features (i.e., spatial extension, density and related asymmetries, orientation and temperature) are connected to solar activity. Therefore in this work two LAD data sets were collected and analyzed separately for two periods with very different solar activity. While the solar minimum data set considers data from the summer solstices 2008 and 2010, the solar maximum data set includes data between 20 October and 24 December 2012.

\section{Coordinate system}

As already described in Zoennchen et al. (2013) we use standard GSE coordinates to fit the neutral exospheric hydrogen distribution with geocentric distance $r$ in $R_{\mathrm{e}}$. The $X-Y$ plane is equal to the ecliptic plane, and the $Z$ axis points towards the ecliptic north pole. The longitudinal angle $\phi$ is counted from $0^{\circ}$ (solar direction) over dusk $\left(90^{\circ}\right)$ to the antisolar direction $\left(180^{\circ}\right)$ and dawn $\left(270^{\circ}\right)$. The latitudinal angle $\theta$ is counted from the $Z$ axis $\left(0^{\circ}\right.$ at the ecliptic north pole) to $180^{\circ}$ (at the ecliptic south pole).

\section{Correction for geocoronal Lyman- $\alpha$ multiple-scattering effects}

The neutral exosphere itself modifies the incoming solar Lyman- $\alpha$ radiation due to multiple-scattering effects. As a result, the local Lyman- $\alpha$ illumination at a given exospheric point $r$ can be significantly influenced by geocoronal Lyman$\alpha$ self-absorption and additionally by Lyman- $\alpha$ re-emission from the lower exosphere. Therefore the resonant scattering rate ( $g$ factor) of Lyman- $\alpha$ photons within the exosphere is a function of the local exospheric position $(g=g(r, \theta, \phi))$. This has to be considered even if the resonant scattering process itself takes place within the optically thin regime of the exosphere ( $\geq 3 R_{\mathrm{e}}$, which is the case in this analysis).

The effect of geocoronal Lyman- $\alpha$ self-absorption is visible as reduced solar Lyman- $\alpha$ illumination of the exosphere on the Earth's nightside, most significant within the penumbra region. On their way from the dayside towards the nightside through the exosphere, solar Lyman- $\alpha$ photons can penetrate optically thick exospheric regions lower than $3 R_{\mathrm{e}}$. For those photons the optical depth $\tau$ increases above 1 significantly, and those solar Lyman- $\alpha$ photons are depleted by exospheric absorption on their way to the nightside. This effect increases with decreasing Earth impact parameter of the solar Lyman- $\alpha$ photons.

The simultaneous multiple-scattering effect is the geocoronal Lyman- $\alpha$ re-emission, which leads to re-emission from the very low (and optically thick) exosphere. Here the $\mathrm{H}$ densities are very high and the medium is very optically

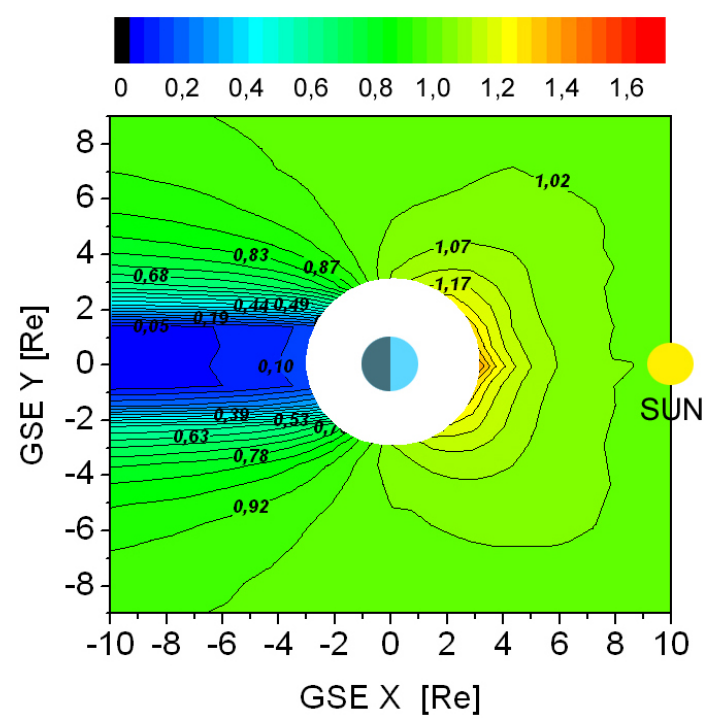

Figure 1. Ratio $\epsilon(r, \theta, \phi)$ of the local Lyman- $\alpha$ illumination (influenced by multiple-scattering effects) and the original solar illumination within the ecliptic plane calculated with a multiple-scattering Monte Carlo model. The exospheric density used in the MCM is taken from Zoennchen et al. (2013). Far from the Earth-Sun line, the exosphere is optically thin for solar Lyman- $\alpha$ photons.

thick $(\tau \gg 1)$. Due to the dominance of multiple scattering in this regime, the inner exosphere acts as an additional source of Lyman- $\alpha$ radiation. Multiple scattering is most effective on the Earth dayside, which is in some sense an additional source of illumination by re-emitted geocoronal Lyman- $\alpha$ photons besides the direct solar radiation.

To be able to correct the TWINS LAD data for both selfabsorption and re-emission, we built a multiple-scattering Monte Carlo model (MCM) based on "coherent line-center scattering" of Lyman- $\alpha$ photons. This model tracks the exospheric trajectories of incoming solar Lyman- $\alpha$ photons from the dayside at $10 R_{\mathrm{e}}$ under multiple-scattering conditions. It can be used with a given H-density distribution $N(r, \theta, \phi)$, which is valid between $1000 \mathrm{~km}$ altitude and $10 R_{\mathrm{e}}$ geocentric distance. Of photons that penetrate the lower model boundary $(1000 \mathrm{~km}$ altitude), $50 \%$ are absorbed and $50 \%$ are backscattered. Photons crossing the upper model boundary at $10 R_{\mathrm{e}}$ are removed. The model considers the angular probability relation given by the scattering function (Eq. 4) between pre- and post-scatter fly directions of the photons.

Using this MCM at a given point $r$ and for a given exospheric density $N(r, \theta, \phi)$ we can calculate values of geocoronal Lyman- $\alpha$ self-absorption and re-emission separately. Both effects need to be incorporated to take into account the effective local Lyman- $\alpha$ illumination.

Based on the averaged $N(r)$ density profile from Zoennchen et al. (2013), Fig. 1 shows the modeled ratio $\epsilon(r, \theta, \phi)$ of the local Lyman- $\alpha$ illumination (influenced by multiplescattering effects) versus the original solar illumination in the 
ecliptic plane. The white circle at $3 R_{\mathrm{e}}$ marks the lower border for the H-density model fits in this work (see Sect. 13 and following). It is evident from Fig. 1 that, far from the Earth-Sun line, i.e., for $\sqrt{Y^{2}+Z^{2}}>3 R_{\mathrm{e}}$, the Lyman- $\alpha$ illumination is (nearly) unchanged from the original solar flux, i.e., $\epsilon \approx 1$. On the dayside and close to the Earth, the re-emission of Lyman- $\alpha$ photons is significant $(\epsilon>1)$ and acts as a second photon source. Therefore, lower regions of the exosphere on the dayside exhibit an enhanced Lyman- $\alpha$ glow. On the nightside (particularly in the penumbra region) the self-absorption effect of Lyman- $\alpha$ photons is pronounced $(\epsilon<1)$. As a consequence, the exosphere on the nightside appears darkened in Lyman- $\alpha$.

The MCM is incorporated in our 3-D H-density modeling in an iterative way, as follows. First, the multiple-scattering effects are calculated for an initial $N(r)$ model (i.e., the averaged $N(r)$ profile from Zoennchen et al., 2013). Second, the ratio $\epsilon(r, \theta, \phi)$ is used to correct the 3 -D H-density fit based on TWINS LAD data, which results in a new $N(r, \theta, \phi)$ model. Since we analyze LAD data above $3 R_{\mathrm{e}}$ only, the corrected density model is extended to lower altitudes by fitting an adapted Chamberlain-like model at $3 R_{\mathrm{e}}$ (Zoennchen et al., 2013). This extension uses a fixed $\mathrm{H}$ density of $50000 \mathrm{~cm}^{-3}$ at $1000 \mathrm{~km}$ (Carruthers et al., 1976) and the particular averaged $\mathrm{H}$ density of the LOS-constrained model at $3 R_{\mathrm{e}}$ to fit a radial symmetric exponential function in between. This extended H-density model is used by the MCM to calculate the next iteration's multiple-scattering effects. This procedure is repeated until two consecutive H-density model iterations (used in the MCM and in the 3-D H-density fit) converge. Convergence is reached if the globally averaged relative error between the density models found in iteration $i$ and $(i-1)$ differs less than $3 \%$ (typically the case after four iterations).

From test variations of the MCM lower boundary (i.e in altitude $-37 \%$ and in $\mathrm{H}$ density $+20 \%$, respectively) we found influences to the density fits above $3 R_{\mathrm{e}}$ smaller than $1 \%$. We conclude that changes in these two parameters within reasonable values do not effect the presented LOSconstrained H-density distributions significantly.

Particularly for the density analysis on the nightside of the Earth, the local $\epsilon$ values need to be considered. As an example, Fig. 2 presents the calculated Lyman- $\alpha$ column brightness (in the GSE $X-Z$ plane) of the terrestrial exosphere as seen by a distant observer. The brightness was calculated based on $\mathrm{H}$ densities of our $N(r, \theta, \phi)$ model for solar minimum (see Sect. 13). In Fig. 2a the correction for the local $\epsilon$ values is included, whereas in Fig. $2 b$ no correction was performed. By comparing these with the Apollo 16 image (Fig. 2c) of the exospheric Lyman- $\alpha$ column brightness as seen from the Moon (Carruthers et al., 1976), it is obvious that the depleted Lyman- $\alpha$ glow on the Earth nightside as pictured by Apollo 16 agrees qualitatively very well in shape with the calculated glow, which takes the geocoronal
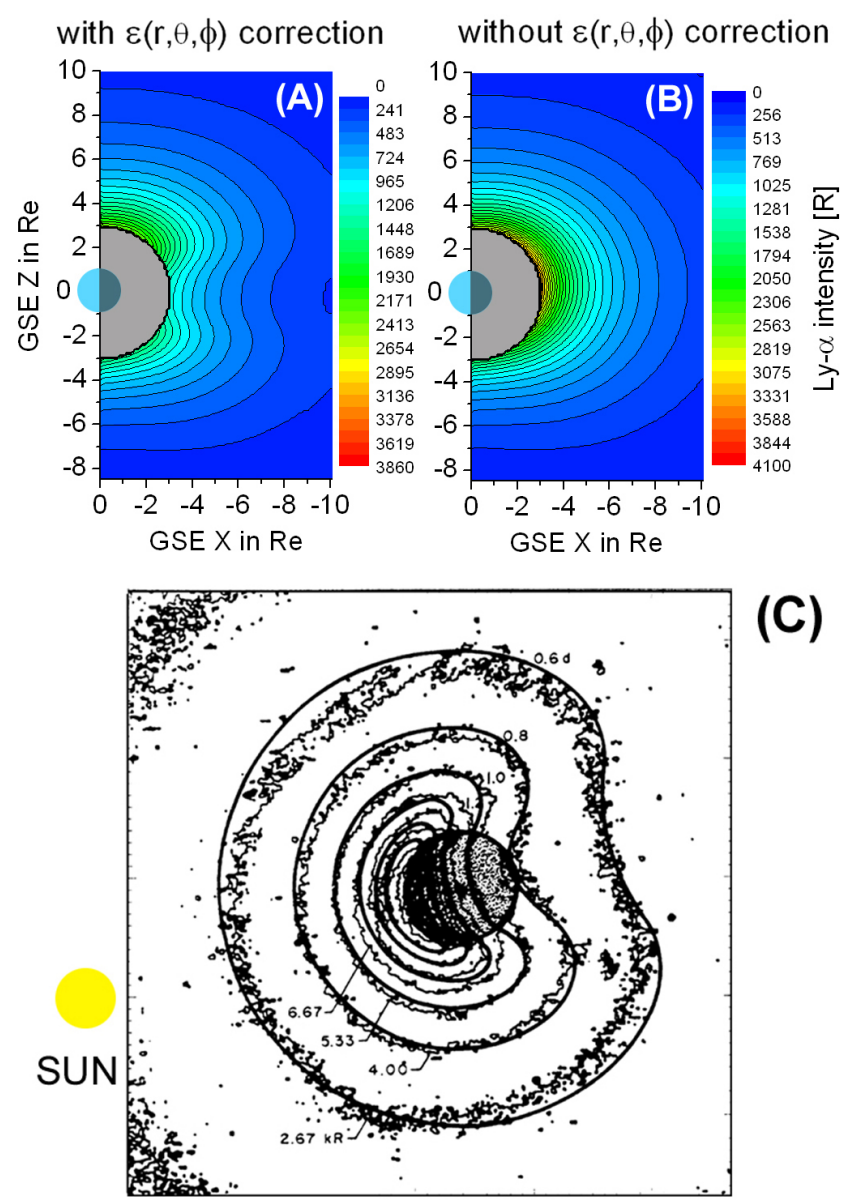

(C)

Figure 2. Calculated and photographed Lyman- $\alpha$ column brightnesses of the terrestrial exosphere. The image in (a) (in units of $[R]$ )) was calculated above $3 R_{\mathrm{e}}$ taking into account the change in the local Lyman- $\alpha$ illumination caused by geocoronal multiple-scattering effects. The image in (b) is similar to (a) but without the multiplescattering correction. Image (c) shows the Lyman- $\alpha$ column brightness in $[\mathrm{kR}]$ as photographed by Apollo 16 from the Moon (taken from Carruthers et al., 1976). The observed shape of the emission as seen from the Moon is in qualitative agreement with the calculated shape in image (a).

multiple-scattering effects into account $(\epsilon<1$ values on the nightside) as shown in Fig. 2a.

In addition to the local $\epsilon$ correction applied in our LAD data analysis, we also exclude LOSs that cross regions of $\epsilon<0.55$. When integrating the LOS intensity numerically, this exclusion avoids nonuniform field-of-view illumination along the LOS. Since the regions between $\epsilon=0.55$ and the Earth core shadow $\epsilon<0.1$ have a very strong Lyman- $\alpha$ illumination gradient over small distances (see Fig. 1), those gradients can appear within the LAD field of view and cause a systematic error. 


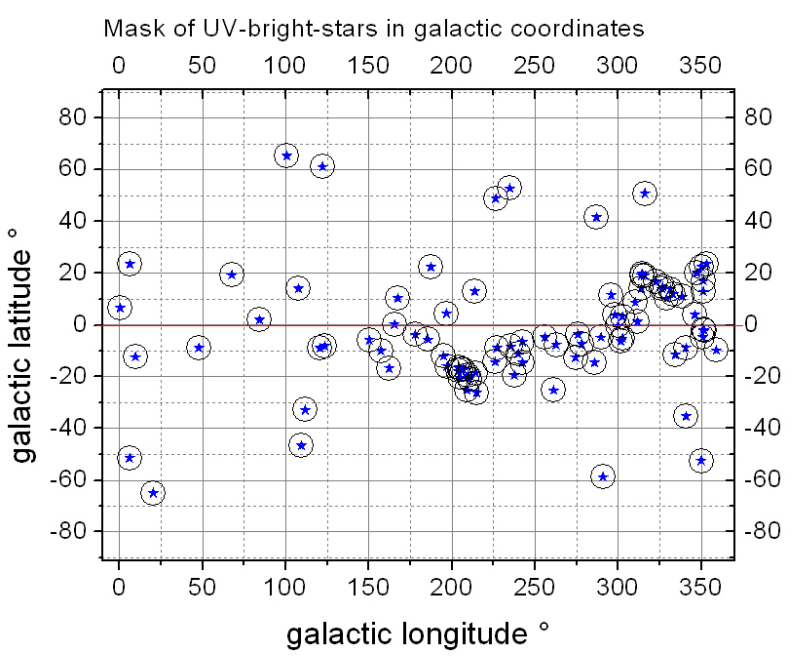

Figure 3. All-sky stellar exclusion map of 90 UV-bright stars based on a list published by Snow et al. (2013). All stars are masked with an $8^{\circ}$ circle for LOS removal.

\section{Correction for stellar UV contamination}

Occasionally, especially for LOSs toward the galactic plane, the LAD LOS crosses stars with a bright UV radiation component (e.g., young O-type stars). These LOSs are thus contaminated by stellar UV radiation and need to be removed from the geocoronal analysis. To exclude stellar UV sources we have created an all-sky map of 90 UV-bright stars as published in Snow et al. (2013), in which these stars are masked with an $8^{\circ}$ circle (see Fig. 3). All LOSs with an intersection of at least one of those circles were removed from the final analysis. The $8^{\circ}$ angular size of an individual star mask was chosen based on the LAD instrument response. During the ground-based LAD calibration at the BESSY synchrotron, it was determined that off-axis Lyman- $\alpha$ intensity from outside a $4^{\circ}$ cone is reduced to $\leq 5 \%$.

On the other hand, the UV brightness of a star that is relatively time-invariant (and a point-like source) can be a very useful source for in-flight, absolute recalibration of the TWINS LAD sensitivities. As described in Sect. 8, we measured the effective LAD sensitivities for the periods between 20 October and 24 December 2012 based on stellar UV intensities.

\section{Correction for interplanetary Lyman- $\alpha$ background}

Scattered Lyman- $\alpha$ radiation by the interplanetary hydrogen density distribution produces a background signal that must be considered in our model. In order to calculate this background signal, we employ some models and assumptions. We use a hot model of the interplanetary density distribution (Fahr, 1971; Thomas, 1978). For the incoming interstel- lar flow we use ecliptic longitude $250.5^{\circ}$ and ecliptic latitude $8.8^{\circ}$.

The bulk velocity and the temperature are assumed to be $v_{0}=22 \mathrm{~km} \mathrm{~s}^{-1}$ and $T=12000 \mathrm{~K}$, respectively (Costa et al., 1999). The density at the termination shock of $n_{0}=0.1 \mathrm{~cm}^{-3}$ is adopted from Bzowski et al. (2008). To take into account the solar influence on the nearby hydrogen density distribution, we use existing databases, as follows. In order to calculate the ionization rate against charge exchange at the solar equator $\beta_{0}$, we use the solar wind proton density and velocity as published in the OMNI database (http://cohoweb.gsfc. nasa.gov/form/omni_m.html).

The needed charge exchange cross section is taken from Bzowski et al. (2001). The obtained ionization rates are then averaged over a period of 1 year. The latitudinal dependence of this ionization rate is also considered assuming a model proposed by Kyrölä et al. (1998):

$$
\begin{gathered}
\beta(\theta)=\beta_{0}\left[\Theta(\theta)\left(1-A_{N} \sin ^{2} \theta\right)+\right. \\
\left.\Theta(-\theta)\left(1-A_{S} \sin ^{2} \theta\right)+B(\theta)\right] .
\end{gathered}
$$

Here $\theta$ is the solar heliographic latitude, $\Theta$ is a step function restricting either of the first two terms to either the Northern or Southern Hemisphere only; $A_{N}$ and $A_{S}$ are the traditional asymmetry parameters (Witt et al., 1979), but individually for both solar hemispheres; and the last factor, $B$, allows for a narrow ionization bulge around the solar equator. The origin of this feature goes back to the Prognoz Lyman$\alpha$ data where a small dip around the ecliptic was observed (Bertaux et al., 1988).

For modeling this feature we again use a formula from Kyrölä et al. (1998):

$B\left(\theta, b_{e}, b_{\mathrm{w}}\right)=b_{e} \exp \left(-\theta^{2} / b_{\mathrm{w}}^{2}\right)$.

The parameter $b_{e}$ gives the strength of this small bulge, and $b_{\mathrm{w}}$ characterizes the width of this feature. The asymmetry parameters $A_{N}$ and $A_{S}$ are taken from Sokól et al. (2013). The ecliptic longitude of the ascending node of the solar equator (J2000) is $75.77^{\circ}$. The inclination of the solar equator to the ecliptic is $7.25^{\circ}$.

To calculate the EUV ionization rates we use $F 10.7 \mathrm{~cm}$ measurements from the UK Solar System Data Centre (http: //www.wdc.rl.ac.uk), averaged over one solar rotation period, and with the aid of a proxy correlation function (Bzowski et al., 2013). The ratio between the solar radiation pressure force and the gravitational force is calculated using the solar Lyman- $\alpha$ line-center intensity. The total solar Lyman- $\alpha$ flux is taken from the LASP (Laboratory for Atmospheric and Space Physics, University of Boulder, Colorado) database. After averaging these data over one solar rotation period, we use the proxy function given by Emerich et al. (2005).

Two examples of the calculated interplanetary Lyman- $\alpha$ background intensities (20 June 2008 for solar minimum and 7 November 2012 for solar maximum) are compared to the 
SOHO/SWAN data in Fig. 4a and b. We estimate the maximum relative error between the SOHO/SWAN data and our model to be around $10 \%$. This estimation is an averaged result by comparison of selected points in the color-coded maps (Fig. 4a and b) of our model and the SOHO/SWAN data.

From test fits of the 3-D number density distribution under a globally $10 \%$ increased interplanetary Lyman- $\alpha$ background, we found that the angularly averaged number density profile is effected by the higher background by about $1 \%$ at $3 R_{\mathrm{e}}$ and about $4 \%$ at $8 R_{\mathrm{e}}$.

\section{Correction for LAD sensitivity variations}

The sensitivity of the LADs change with time caused by aging, thermal or other effects. To compensate for the loss of sensitivity, the high voltage of the LAD photo multipliers was twice adjusted to higher values, on 21 March 2011 and 4 October 2012. With the exception of TWINS 1 LAD data between June and September 2008 (see reference period in Zoennchen et al., 2013), all other TWINS LAD data need to be recalibrated with respect to the sensitivity of their observation date.

One effective way of measuring the absolute LAD sensitivity is the observation of stars with a bright UV radiation component and no (or low) time variability. Stars appear within the data as very sharp intensity peaks on top of the smooth exospheric and interplanetary Lyman- $\alpha$ emission. Therefore the star intensities can be isolated using a highpass filter (performed in frequency space), which removes the slowly varying exospheric and interplanetary Lyman- $\alpha$ components.

The presented star calibration method is based on TWINS LAD data with their highest time resolution $(0.67 \mathrm{~s}$ per measurement). For every presented star we used a large number of stellar field-of-view crossings, which allow a statistical judgement. First, we found a strong, significant increase in the LAD count rate for decreasing angular distances $\left(\leq 4^{\circ}\right)$ between the LOS direction and the stellar position. Additionally, we found that the LAD count rate and the angular distance $\left(\leq 4^{\circ}\right)$ are strongly correlated. Pointing uncertainties (e.g., caused by the LOS movement during the $0.67 \mathrm{~s}$ data accumulation time) cause statistical count-rate fluctuations for identical angular distances. Those fluctuations were found to be much smaller compared to the significant angularly related increase in count rates as mentioned above (i.e., the peak emission of 3700 counts per $0.67 \mathrm{~s}$ for star $\alpha^{1,2} \mathrm{Cru}$ in the middle-left panel of Fig. 5 has a signal-to-noise ratio of about 10). Since some stars have a real or a projected "nearby companion" star in the sky, the statistical fluctuations with angular distance can be reduced by analyzing the relation between the LAD counts and the two-dimensional angular distance (taking into account separately the angular distance in both galactic longitude and latitude).
TWINS 1 LAD2 observed the two stars Spica ( $\alpha$ Vir) and $\alpha^{1,2}$ Cru (part of the Southern Cross) with wellknown sensitivity factors within the reference period JuneSeptember 2008 (Fig. 5, top-left and middle-left panels). At this time the ground-based calibration was still valid for TWINS 1 . These two stars were observed again by TWINS 1 LAD1 during spring equinox 2010 (see Fig. 5, top-right and middle-right panels). After isolating the stellar emission and by comparison of the (same stars) peak level for 2008 and 2010, the sensitivity of LAD1 (20 March 2010) was found to be approximately 0.95 counts $\mathrm{s}^{-1} \mathrm{R}^{-1}$. This is in good agreement with the value of 1.03 counts s ${ }^{-1} \mathrm{R}^{-1}$, which was found based on a different method using the geocoronal glow under constant solar conditions as a calibration source (Zoennchen et al., 2013). For TWINS 2 LAD data (20 June 2008) we therefore continue to use the calibration factors presented in Zoennchen et al. (2013).

The star $\gamma^{2}$ Vel was used to calibrate the sensitivity of TWINS 1 LAD1 in 2012. This star was observed at the spring equinox 2010 by TWINS 1 LAD2 and again during 20-24 December 2012 by TWINS 1 LAD1 (Fig. 5, bottom panels). Using the same method the sensitivity of LAD1 (20-24 December 2012) was found to be approximately 1.55 counts $\mathrm{s}^{-1} \mathrm{R}^{-1}$. We validated these calibration values with factors found independently by comparing the complete distributed stellar intensities (pixel by pixel) of the corresponding left and right images in Fig. 5. Results of both methods differ very slightly (Spica $1.35 \%, \alpha^{1,2}$ Cru $0.78 \%$ and $\gamma^{2} \mathrm{Vel} 0.28 \%$ ). From the statistical fluctuations within the compared images we estimate the uncertainty of the stellar calibration to be about 7-10\%.

If the sensitivity of just one of the two LADs is known (i.e., based on a star recalibration), the unknown one can be found using the method of relative recalibration as discussed in Zoennchen et al. (2013). This method is based on the fact that both LADs have (nearly) overlapping view directions at the actuators turning points, which should (in principle) be observed with the same intensity by both detectors.

\section{Scattering phase function correction}

There is an angular intensity dependence $I_{\mathrm{p}}(\alpha)$ of the scattered Lyman- $\alpha$ photons with respect to the direction of the incoming (solar) Lyman- $\alpha$ photons as described by Brandt and Chamberlain (1959):

$I_{\mathrm{p}}(\alpha)=1+\frac{1}{4}\left(\frac{2}{3}-\sin ^{2}(\alpha)\right)$

A detailed physical discussion of the scattering function in Eq. (4) can be found in Brasken and Kyrölä (1998). This scattering function $I_{\mathrm{p}}$ has two maxima (at $\alpha=0^{\circ}, 180^{\circ}$ ) and two minima (at $\alpha=90^{\circ}, 270^{\circ}$ ). At the maxima, $I_{\mathrm{p}}$ contributes $+16.7 \%$. At the minima, $I_{\mathrm{p}}$ is a reduction of $-8.3 \%$. Therefore resonant Lyman- $\alpha$ scattering has a higher probability 
(a)
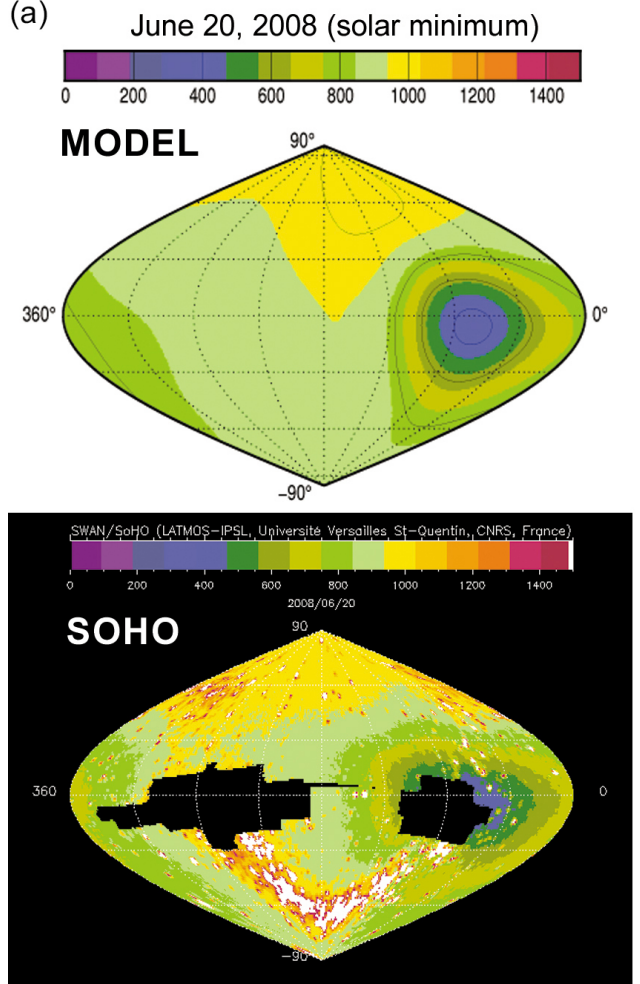

(b) Nov. 07, 2012 (near solar maximum)
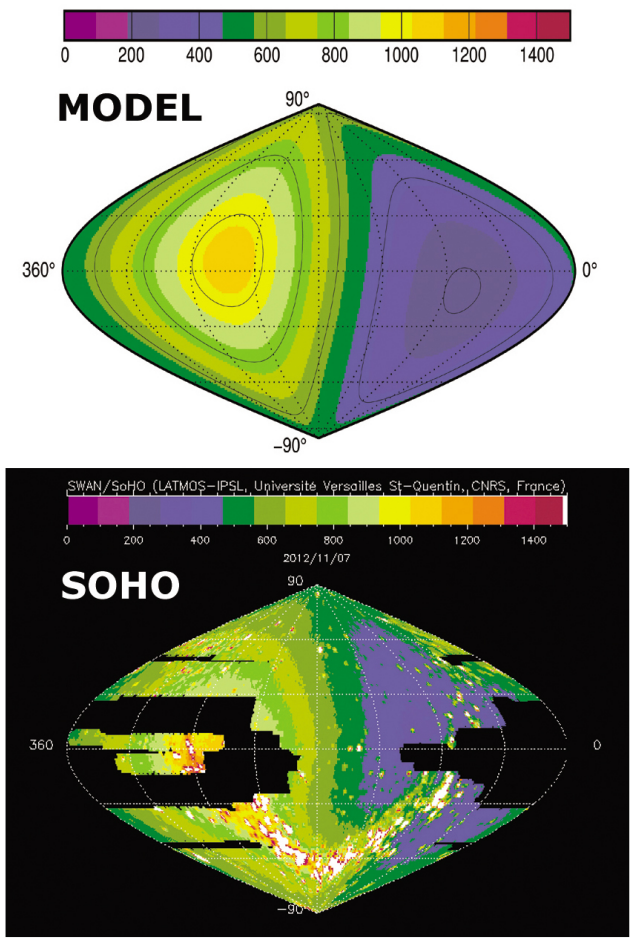

Figure 4. (a) Solar minimum all-sky maps for interplanetary Lyman- $\alpha$ background intensities [R] for 20 June 2008. Top: our simulated hot-model Lyman- $\alpha$ intensity map using $A_{N}=A_{S}=0.4, b_{e}=0.04$, and $b_{\mathrm{w}}=11.5^{\circ}$. Bottom: observed Lyman- $\alpha$ all-sky intensities by SOHO/SWAN (provided via Web by LATMOS-IPSL, Université Versailles St-Quentin, CNRS, France: http://swan.projet.latmos.ipsl.fr/ images/). (b) Near-solar-maximum all-sky maps for interplanetary Lyman- $\alpha$ background intensities [R] for 7 November 2012. Top: our simulated hot-model Lyman- $\alpha$ intensity map using $A_{N}=A_{S}=b_{e}=0$. Bottom: observed Lyman- $\alpha$ all-sky intensities by SOHO/SWAN.

of forward or backward scattering compared to the isotropic case. For regions perpendicular to pre-scatter velocity direction, the probability is slightly lower. The quantitative influence of the scattering function to the global number density fit depends on the concrete observational geometry. In the density model fits, the effect of the scattering function is considered with the $I_{\mathrm{p}}(\alpha(S))$ term in the numerical integration of the column brightness along the LOS as shown in Eq. (1).

The $I_{\mathrm{p}}$-value of the direct solar Lyman- $\alpha$ intensity at a given LOS point $\mathrm{d} S$ can be calculated using the angle $\alpha$ between the (inverse) LOS direction and solar direction. For the indirect Earth re-emitted Lyman- $\alpha$ intensity we calculated the $I_{\mathrm{p}}$-value based on the approximation that, above $3 R_{\mathrm{e}}$, the majority of re-emitted photons are coming from the Earth direction (angle $\alpha$ between inverse LOS direction and Earth direction at $d \mathrm{~S}$ ). For relevant LOSs, the indirect Lyman- $\alpha$ intensity typically contributes $2-10 \%$ only to the total Lyman$\alpha$ illumination at a given point $\mathrm{d} S$. Taking into account additionally the small deviation of the scattering function from the isotropic case (Eq. 4), we can estimate the total uncertainty of this approximation for the density fits to be $\ll 1 \%$.

\section{Solar activity conditions}

Total solar Lyman- $\alpha$ flux is strongly correlated with solar activity. The total solar Lyman- $\alpha$ flux is measured by TIMED SEE and SORCE SOLSTICE, calibrated to the UARS SOLSTICE level (Woods et al., 2000) (provided by LASP). The total solar Lyman- $\alpha$ flux is plotted in Fig. 6 for the periods we have analyzed.

The observations during solar minimum at the summer solstices 2008 and 2010 (Fig. 6, top) were taken under stable solar activity at a very low level. In late 2009 , the earliest sign of the upcoming end of the minimum of solar cycle 23 was evident as a slow, $<9 \%$ increase in the the 27 -dayaveraged solar Lyman- $\alpha$ flux. During this period the variation on timescales shorter than 27 days was less than or comparable to the long-timescale increase.

The situation is completely different for the observations from near-solar-maximum conditions, taken between 20 October and 24 December 2012. During that period the solar activity level fluctuated significantly (20\% to $30 \%$ ) within each 27-day solar rotation interval (Fig. 6, bottom). The 27day-averaged total solar Lyman- $\alpha$ flux decreased continuously by $<10 \%$ (Fig. 6 , bottom, cyan line) between point 

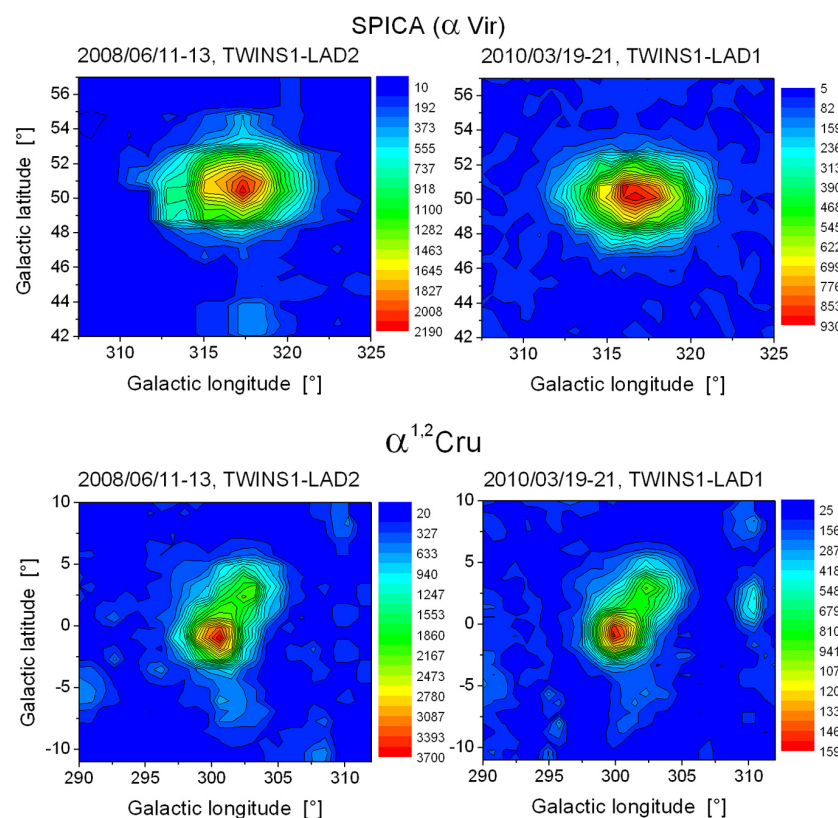

$\alpha^{1,2}$ Cru
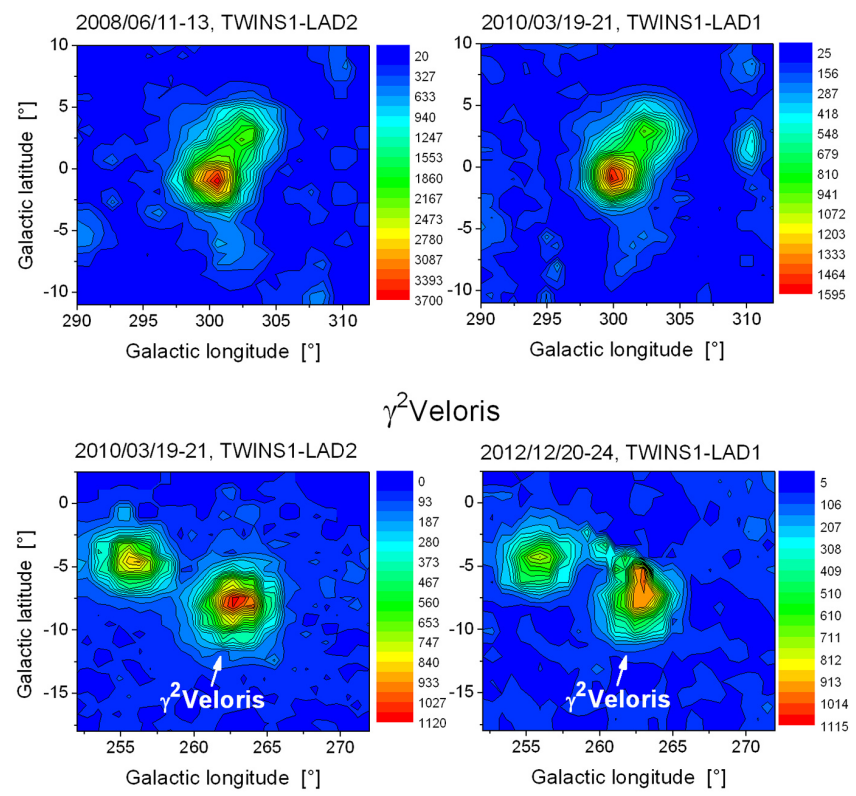

$\gamma^{2}$ Veloris

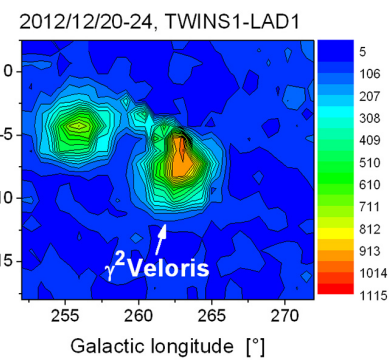

Figure 5. TWINS 1 LAD observations of stellar UV emission in [counts/0.67 s] from UV-bright stars (separated from exospheric and interplanetary emission): Spica ( $\alpha$ Vir) in 2008 and 2010 (top), $\alpha^{1,2}$ Cru in 2008 and 2010 (middle), and $\gamma^{2}$ Vel in 2010 and 2012 (bottom).

1 and 4. For comparison, the averaged 27-day total solar Lyman- $\alpha$ flux at 20 October 2012 (near solar maximum) was $26 \%$ higher than its value at summer solstice 2008 (solar minimum). The daily fluctuating $g$ factors caused by the different solar Lyman- $\alpha$ fluxes were considered in our density calculations.

Bailey and Gruntman (2013) reported an effect of disturbed magnetospheric conditions to the exospheric number density of about $5 \%$ for Dst $=-70 \mathrm{nT}(10 \%$ for Dst $=$ $-105 \mathrm{nT})$. The effect increases with falling Dst values. For all periods we have used in our analysis, the Dst values were stable and not lower than $-25 \mathrm{nT}$ (20 December 2012 at 16:00 UT). Therefore we did not take this effect into account in the presented fits.

In relation to previous solar maxima (i.e., around 2001), the current solar cycle 24 maximum was unusually small (McComas et al., 2013). Therefore, the solar Lyman- $\alpha$ flux total solar Ly- $\alpha$ flux: June 2008 - Aug. 2010

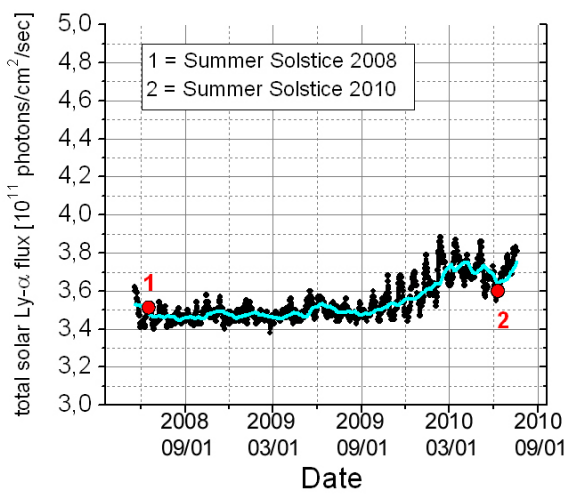

total solar Ly- $\alpha$ flux: Oct. - Dec. 2012

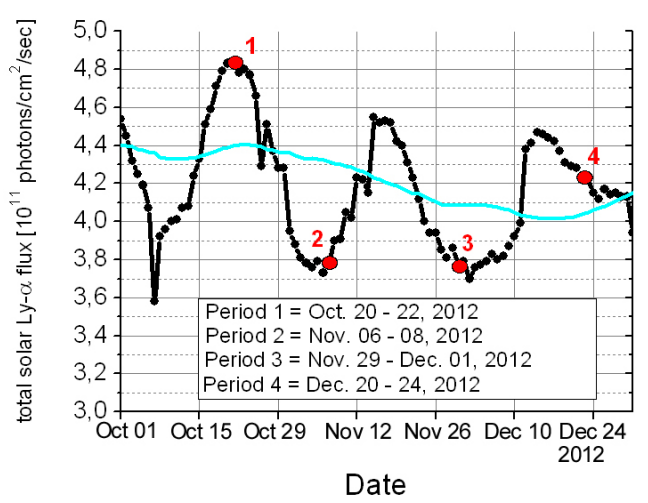

Figure 6. Total solar Lyman- $\alpha$ flux for the period of the solar minimum data set (top) and the solar maximum data set (bottom). The cyan lines show the 27-day average of the flux.

of the current maximum is comparable to solar medium conditions in a more typical solar cycle.

\section{Observational coverage and raw data}

The quality of a global 3-D density fit is critically influenced by the observational coverage of the circumterrestrial exospheric space. The two TWINS spacecraft change their solar orientation continuously while orbiting together with the Earth around the Sun, and this provides a periodically varying coverage. For example, a $90^{\circ}$ solar orientation shift within 3 months opens up very different geocoronal regions for TWINS LAD observations and increases the observational coverage significantly.

To characterize the dependence on solar activity, we collected TWINS LAD observations in two separate data sets. The first data set is for solar minimum conditions, obtained from summer solstice 19-21 June 2008 (TWINS 1 and 2) and 19-21 June 2010 (TWINS 1). The second data set is for solar maximum conditions and is composed of four periods in 

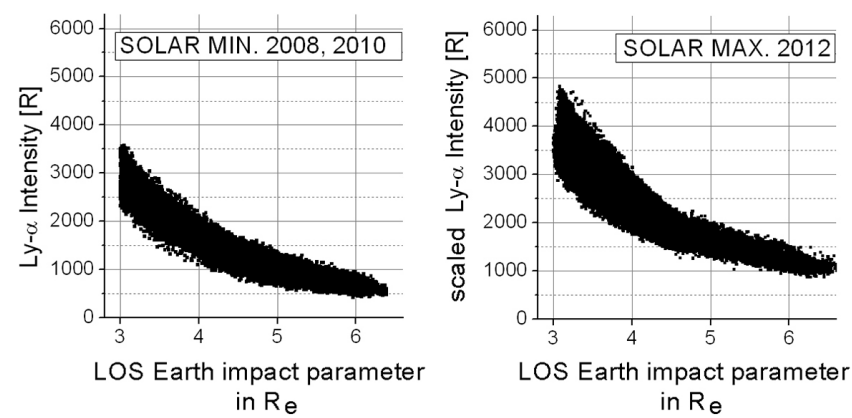

Figure 7. LOS Lyman- $\alpha$ intensities against their Earth impact parameters. Intensities on the right figure (solar maximum) are plotted scaled with the ratio of solar Lyman- $\alpha$ fluxes at 20 June 2008 and at each specific period as expressed in Eq. (5).

2012: 20-22 October; 6-8 November, 29 November-1 December; 20-24 December (TWINS 1).

In Fig. 7 the observed geocoronal Lyman- $\alpha$ intensities (absolute recalibrated and corrected for interplanetary Lyman- $\alpha$ background) are presented for both data sets. The LOS intensities are plotted against their Earth impact parameter. To better clarify the purely density-driven intensity differences between the left and right panels of Fig. 7, the increased intensity and scattering caused by the enhanced and strongly varying solar Lyman- $\alpha$ fluxes during the solar maximum periods need to be removed. Therefore the intensities $I$ of each of the four periods during solar maximum (right panel) are plotted scaled with the ratio at the solar minimum Lyman- $\alpha$ fluxes of 20 June 2008 and at each specific period:

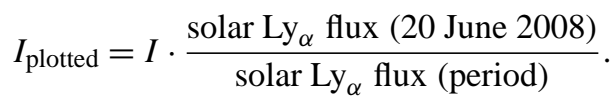

Overall we collected $\approx 46000$ observations for the solar minimum data set and $\approx 98000$ observations for the solar maximum data set. The observational coverage in the data sets is presented for the ecliptic plane and the polarmeridional plane in Figs. 8 and 9.

Due to a systematic error most of the dayside coverage is lost (for angles between the LOS direction and the Sun $\leq 90^{\circ}$ ) because of a direct instrumental stray-light reflection of solar Lyman- $\alpha$ radiation to the detectors. Unfortunately this reflection problem removes most of the dayside LOSs whose closest approach to Earth is $>5 R_{\mathrm{e}}$.

Another systematic issue is the difference of the coverage quality between the Northern and the Southern Hemisphere caused by the TWINS mission geometry. Since the TWINS satellites observe from the Northern Hemisphere towards south only, the northern coverage (particular near the Earth's North Pole region) is more complete than the southern coverage.
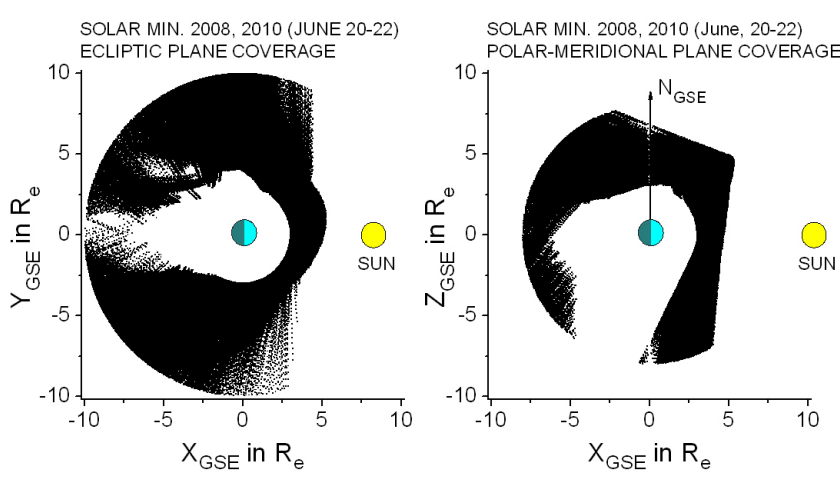

Figure 8. Observational LOS coverage of the solar minimum data set: LOS intersections with (left) the ecliptic plane (within an angular extent of $\theta \pm 25^{\circ}$ around the ecliptic plane) and (right) the polar-meridional plane (within an angular extent of $\phi \pm 25^{\circ}$ around the polar-meridional plane).
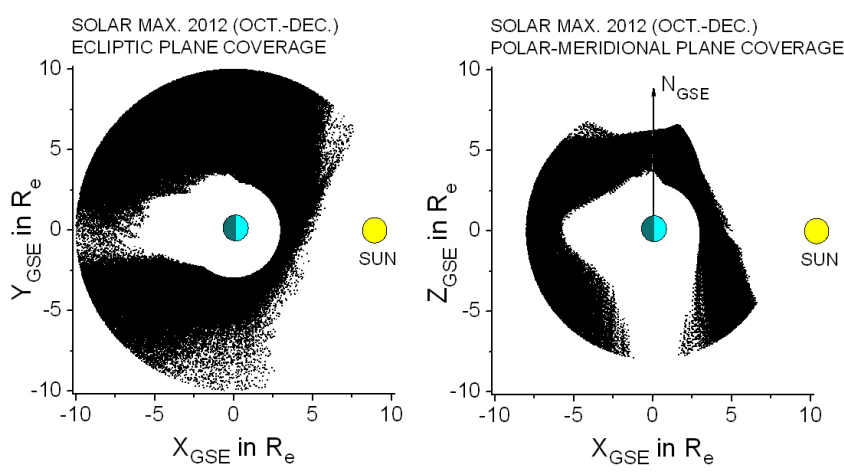

Figure 9. Observational LOS coverage of the solar maximum data set: LOS intersections with (left) the ecliptic plane (within an angular extent of $\theta \pm 25^{\circ}$ around the ecliptic plane) and (right) the polar-meridional plane (within an angular extent of $\phi \pm 25^{\circ}$ around the polar-meridional plane)

\section{Mathematical model of the neutral exosphere}

In this work the mathematical model of the exospheric $\mathrm{H}$ density distribution between 3 and $8 R_{\mathrm{e}}$ (within the optically thin regime) is identical to the model as described in Zoennchen et al. (2013). This means that we use the spherical harmonic representation and the $\mathrm{Y}_{\operatorname{lm}}(\theta)$ terms as presented by Hodges Jr. (1994) up to a polynomial number $l=2$,

$n_{\mathrm{H}}(r, \theta, \phi)=N(r) \sqrt{4 \pi} \sum_{l=0}^{2} \sum_{m=0}^{l} Z(r, \theta, \phi)$,

with

$Z(r, \theta, \phi)=\left[A_{\operatorname{lm}}(r) \cos (m \phi)+B_{\operatorname{lm}}(r) \sin (m \phi)\right] Y_{\operatorname{lm}}(\theta)$,

where $\mathrm{Y}_{\operatorname{lm}}(\theta)$ are the spherical harmonic Legendre polynomials. For the $N(r)$ term we use a power law $N(r)=c \cdot r^{-k}$ function. 
Table 1. Solar minimum 2008 and 2010 model fit coefficients. The $A_{\operatorname{lm}}$ and $B_{\operatorname{lm}}$ coefficients (with the exception of $A_{00}$ ) have to be multiplied by $10^{-4}$.

\begin{tabular}{lll}
\hline$N(r)=c \cdot r^{-k}$ & $c=12264.1 ;$ & $k=2.87646$ \\
\hline$A_{00}(r)=1$ & always & \\
$A_{10}(r)=a_{10}+b_{10} \cdot \ln (r)$ & $a_{10}=938.00 ;$ & $b_{10}=135.41$ \\
$A_{11}(r)=a_{11}+b_{11} \cdot \ln (r)$ & $a_{11}=92.20 ;$ & $b_{11}=198.16$ \\
$A_{20}(r)=a_{20}+b_{20} \cdot \ln (r)$ & $a_{20}=-385.26 ;$ & $b_{20}=-597.06$ \\
$A_{21}(r)=a_{21}+b_{21} \cdot \ln (r)$ & $a_{21}=2042.26 ;$ & $b_{21}=-916.65$ \\
$A_{22}(r)=a_{22}+b_{22} \cdot \ln (r)$ & $a_{22}=-421.34 ;$ & $b_{22}=1196.10$ \\
$B_{11}(r)=p_{11}+q_{11} \cdot \ln (r)$ & $p_{11}=4870.41 ;$ & $q_{11}=-2632.88$ \\
$B_{21}(r)=p_{21}+q_{21} \cdot \ln (r)$ & $p_{21}=-2506.10 ;$ & $q_{21}=1578.28$ \\
$B_{22}(r)=p_{22}+q_{22} \cdot \ln (r)$ & $p_{22}=2783.95 ;$ & $q_{22}=-1331.32$ \\
\hline
\end{tabular}

Again, the $r$ dependence of the $A_{1 \mathrm{~m}}(r)$ and $B_{\operatorname{lm}}(r)$ coefficients was replaced by the natural logarithmic functions

$A_{\operatorname{lm}}=\left(a_{\operatorname{lm}}+b_{\operatorname{lm}} \cdot \ln (r)\right) \times 10^{-4}$

$B_{\operatorname{lm}}=\left(p_{\operatorname{lm}}+q_{\operatorname{lm}} \cdot \ln (r)\right) \times 10^{-4}$,

with constant $A_{00}=1$.

\section{Model fits for solar minimum and solar maximum}

The separate model fits for the solar minimum and solar maximum data sets are presented as contour plots in Fig. 10 (left images: ecliptic cuts; right images: polar-meridional cuts). In addition the corresponding fit coefficients are shown in Tables 1 and 2.

As mentioned in Sect. 7, the $10 \%$ maximal interplanetary Lyman- $\alpha$ uncertainty has an effect on the LOS-constrained number density fit of about $1-4 \%$ between 3 and $8 R_{\mathrm{e}}$. For the star calibration we estimate a global 7-10\% uncertainty. In addition we assume the uncertainties for the mathematical model assumption, the finite number of iterations, and the instrumental noise to be about $5 \%$. Therefore, in total we expect density errors of about 15-20\% between 3 and $8 R_{\mathrm{e}}$.

The most obvious fit results consist in the fact that the 3 D H-density distribution under solar maximum conditions shows enhanced densities between 3 and $8 R_{\mathrm{e}}$ compared to the solar minimum exosphere (see averaged H-density profiles in Fig. 11) and appears (spatially) more extended into circumterrestrial space. This can be understood as the thermal expansion of the exospheric neutral gas due to a larger exospheric temperature resulting from higher solar activity.

Besides the density difference between solar minimum and solar maximum, we also found density reactions caused by solar activity variations on monthly timescales: between 20 October and 24 December 2012 the 27-day average of the total solar Lyman- $\alpha$ flux was decreasing continuously, resulting in an exospheric cooling process for about 2 months. We did $N(r)$ power-law fits for both dates separately and found slightly larger $\mathrm{H}$ densities for 20-22 October compared to 20-24 December 2012 as expected (see Fig. 11).
Solar Minimum Conditions Model 2008, 2010

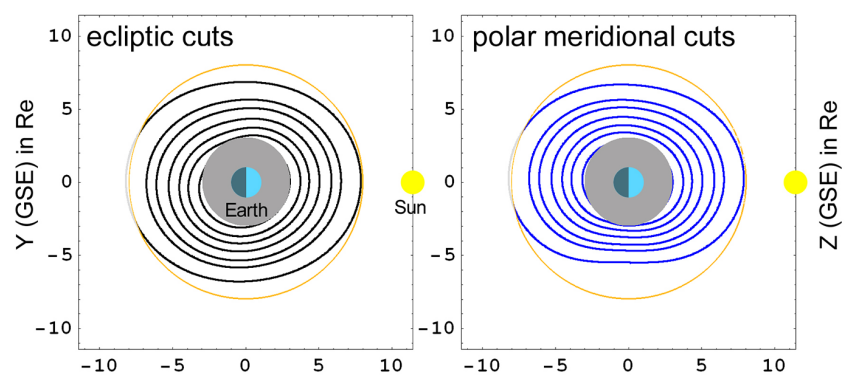

(Near) Solar Maximum Conditions 2012

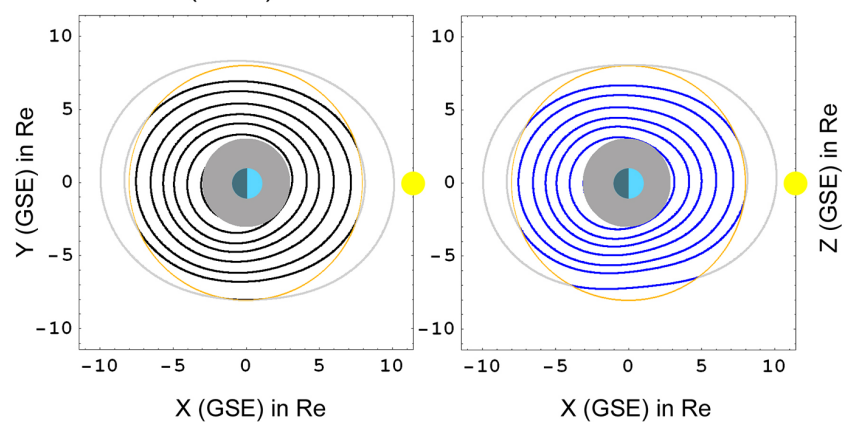

Figure 10. Geocoronal H-density distributions for solar minimum (top) and solar maximum (bottom) as ecliptic cut (left) and polarmeridional cut (right). Density contours for all four images from outside to inside: $45,75,100,150,225,337$ and $600\left[\mathrm{~cm}^{-3}\right]$. The yellow circles at $8 R_{\mathrm{e}}$ indicate the upper validity range of the model. Density contours at larger distances $\left(>8 R_{\mathrm{e}}\right)$ are modelextrapolated and therefore colored light-grey.

Table 2. Near-solar-maximum 2012 model fit coefficients. The $A_{1 \mathrm{~m}}$ and $B_{1 \mathrm{~m}}$ coefficients (with the exception of $A_{00}$ ) have to be multiplied by $10^{-4}$.

\begin{tabular}{lll}
\hline$N(r)=c \cdot r^{-k}$ & $c=16840.9 ;$ & $k=2.74640$ \\
\hline$A_{00}(r)=1$ & always & \\
$A_{10}(r)=a_{10}+b_{10} \cdot \ln (r)$ & $a_{10}=-921.29 ;$ & $b_{10}=790.11$ \\
$A_{11}(r)=a_{11}+b_{11} \cdot \ln (r)$ & $a_{11}=6763.12 ;$ & $b_{11}=-3088.94$ \\
$A_{20}(r)=a_{20}+b_{20} \cdot \ln (r)$ & $a_{20}=-494.96 ;$ & $b_{20}=-405.36$ \\
$A_{21}(r)=a_{21}+b_{21} \cdot \ln (r)$ & $a_{21}=-284.02 ;$ & $b_{21}=44.03$ \\
$A_{22}(r)=a_{22}+b_{22} \cdot \ln (r)$ & $a_{22}=-556.96 ;$ & $b_{22}=1303.13$ \\
$B_{11}(r)=p_{11}+q_{11} \cdot \ln (r)$ & $p_{11}=1289.94 ;$ & $q_{11}=-788.54$ \\
$B_{21}(r)=p_{21}+q_{21} \cdot \ln (r)$ & $p_{21}=-753.84 ;$ & $q_{21}=256.56$ \\
$B_{22}(r)=p_{22}+q_{22} \cdot \ln (r)$ & $p_{22}=2029.43 ;$ & $q_{22}=-1084.30$ \\
\hline
\end{tabular}

However, for (even shorter) solar activity variations on scales of several days we could not detect an instantaneous response of the neutral exosphere. Therefore we assume that exospheric heating or cooling caused by (moderate) solar activity variations have to prevail at least on a monthly timescale to be able to change the outer exospheric densities remarkably.

While the latitudinal structures of the exosphere seem to be correlated with the Earth-Sun line, the ecliptic longitudi- 


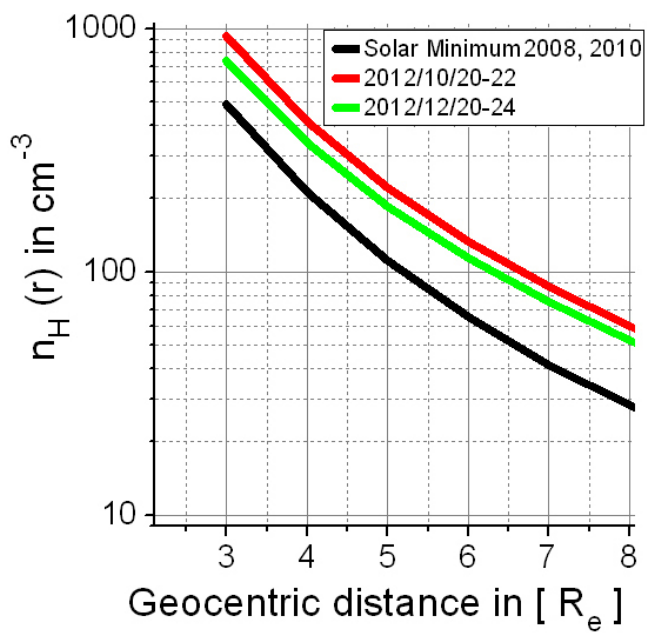

Figure 11. Averaged H-density profiles (power law $N(r)$ ) for solar minimum 2008 and 2010 (black), 20-22 October 2012 (red), and 20-24 December 2012 (green).

nal shape shows some deviations from that orientation, which are distance-dependent (see Sect. 14).

Another interesting result is the detection of a region with enhanced $\mathrm{H}$ densities on the Earth nightside ("H geotail") as discussed in Sect. 15. The feature appears dawn-shifted at lower distances. Finally, values of the dawn-dusk asymmetry and the polar density depletion are discussed in Sects. 16 and 17 , respectively.

\section{Longitudinal H-density variations}

Besides the dominating power-law decrease in the density with distance $r$, also angular dependences are pronounced. The latitudinal variation mainly appears as a polar density depletion and is discussed in Sect. 17.

The longitudinal variation of the $\mathrm{H}$ density is shown in Fig. 12 (normalized to the dusk density $\phi=90^{\circ}$ and for three different distances: $r=3,4.5,8 R_{\mathrm{e}}$ ).

At lower distances $\left(3 R_{\mathrm{e}}\right)$, the dayside density maximum seems to be shifted towards dusk for solar minimum (peak at $\approx 13: 00 \mathrm{LT}$ ). Since the $\mathrm{H}$ atoms need some time to be transported from the lower atmosphere up to the exosphere, this peak of emission should appear with some time delay with respect to noon. For solar maximum we did not find this peak, because here we most likely do not have enough dayside coverage. In distances above $3 R_{\mathrm{e}}$ a lot of the thermally upflowing $\mathrm{H}$ atoms are scattered into elliptic orbits with apogees on the opposite side of the Earth. That scenario explains the existence of a second, post-midnight density peak at $3 R_{\mathrm{e}}$ (02:30 LT solar minimum, 01:00 LT solar maximum).

With increasing distance $r$ the exospheric shape changes its GSE longitudinal orientation continuously to become parallel to the (aberrated) direction of the incoming solar wind.
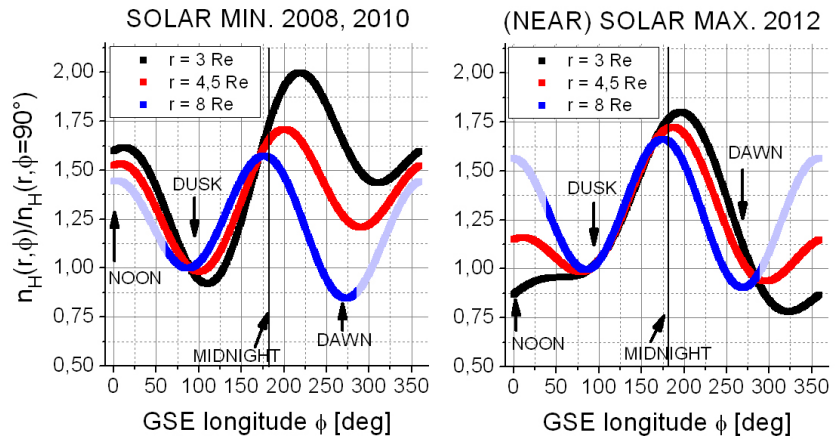

Figure 12. Longitudinal $\mathrm{H}$ density $N(r, \phi)$ normalized to the dusk $\mathrm{H}$ density $N\left(r, \phi=90^{\circ}\right)$ for three distances: $3 R_{\mathrm{e}}$ (black), $4.5 R_{\mathrm{e}}$ (red) and $8 R_{\mathrm{e}}$ (blue). Light-blue indicates missing or bad observational coverage in this region.

Interaction effects which might dominate the shape at lower distances $\left(3 R_{\mathrm{e}}\right)$ seem to lose their relevance and might be replaced by other interactions (i.e., by the solar wind or by particles from the outer magnetosphere). At $8 R_{\mathrm{e}}$ the exosphere (at least the part we can observe from dusk until dawn, including the nightside) is aligned with the direction of the incoming solar wind. Both fits (solar minimum and maximum) have pre-midnight-shifted $\left(-4\right.$ to $-5^{\circ}$ ) density peaks at $8 R_{\mathrm{e}}$, which is equivalent to a small tilt against the Earth-Sun line.

\section{Enhanced H densities at the Earth's nightside (H geotail)}

The $\mathrm{H}$ densities on the Earth's nightside seem to be remarkably enhanced in some particular regions (compared to the dusk H-density profile). This density enhancement is visible in the model fit for solar minimum as well as in the fit for solar maximum. An adequate phenomenon was first mentioned by Thomas and Bohlin (1972) and has since then been referred to as the "H geotail".

Our results suggest a density maximum at low distances ( $3 R_{\mathrm{e}}$ ) that appears post-midnight and is shifted towards the dawn direction (see Fig. 13). At solar minimum this density peak is more strongly dawn-shifted $(\approx 02: 30 \mathrm{LT})$ compared to solar maximum $(\approx 01: 00 \mathrm{LT})$. With increasing distance $r$, and independent of the solar activity, the density maximum is shifted back to midnight and some degrees beyond towards dusk. At $8 R_{\mathrm{e}}$ both model fits show a H-geotail phenomenon aligned parallel to the incoming solar wind direction. We conclude from this alignment that, at $8 R_{\mathrm{e}}$, interactions with particles from outside (i.e., from solar wind) can influence the shape of the tail and its orientation.

Differences in the relative strength and the shape of the $\mathrm{H}$ geotail exist with respect to different solar activities. The $\mathrm{H}$ geotail appears broader and (relative) stronger at $3 R_{\mathrm{e}}$ during solar minimum compared to solar maximum. With increas- 


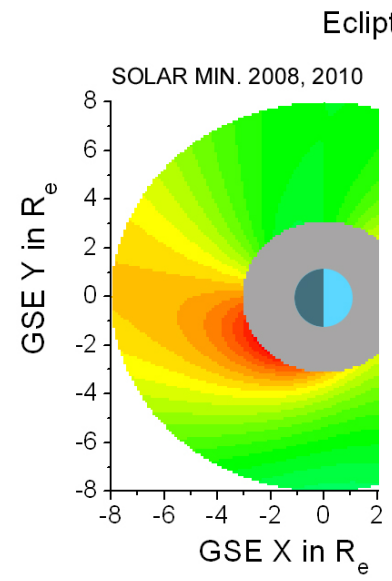

Ecliptic $\mathrm{n}_{\mathrm{H}}(\mathrm{r}, \phi) / \mathrm{n}_{\mathrm{H}}\left(\mathrm{r}, \phi=90^{\circ}\right)$

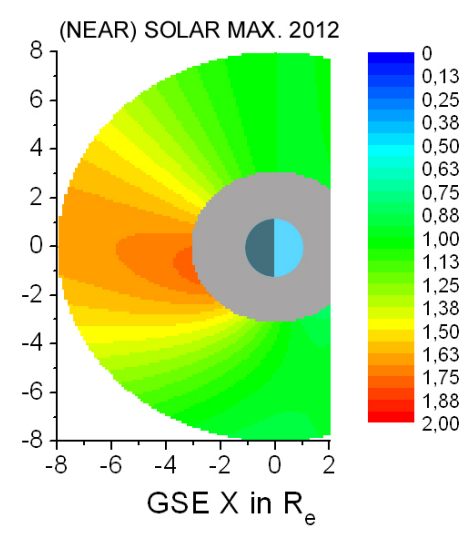

Figure 13. Regions of enhanced $H$ densities at the Earth's nightside within the ecliptic plane. The images show the ratio of the local ecliptic densities $N(r, \phi)$ and the ecliptic dusk density $N(r, \phi=$ $90^{\circ}$ ) for solar minimum (left) and solar maximum (right).

ing distance $r$ the solar minimum tail becomes narrow faster and loses its magnitude faster than at solar maximum.

Figure 13 shows the ratio of the ecliptic densities $N(r, \phi)$ relative to the ecliptic dusk density $N\left(r, \phi=90^{\circ}\right)$ between 3 and $8 R_{\mathrm{e}}$ on the nightside of the Earth. The regions of the enhanced density are clearly visible for solar minimum as well as for solar maximum.

\section{Dawn-dusk asymmetry}

As described for solar minimum in Zoennchen et al. (2013), there is a density asymmetry between ecliptic dusk and dawn with larger densities on the dawn side. The asymmetry vanishes with increasing distance $r$ and turns to higher densities on the dusk side at larger distances. First, in this work we can confirm the value found for the solar minimum. Second, we have also found an analogous but smaller effect for solar maximum. Figure 14 shows the ratio of the H-column densities for the regions between "midnight-dawn" and "midnightdusk".

Considering the H-geotail analysis (see Sect. 15), the dawn-dusk asymmetry seems to be a side effect of the dawnshifted $\mathrm{H}$ geotail at lower distances. Since the solar minimum $\mathrm{H}$ geotail at $3 R_{\mathrm{e}}$ is more dawn-shifted and covers a broader area (compared to solar maximum), the resulting dawn-dusk asymmetry is expected to be more pronounced. With increasing distance $r$ the $\mathrm{H}$ geotail (as possible origin of the dawndusk asymmetry) changes its orientation back from the dawn side towards midnight (and even beyond; see Fig. 13). That explains the decrease in the dawn-dusk asymmetry with increasing distance $r$.

Interestingly, at a particular (larger) distance $r$ the dawndusk asymmetry turns to higher values on the dusk side (as

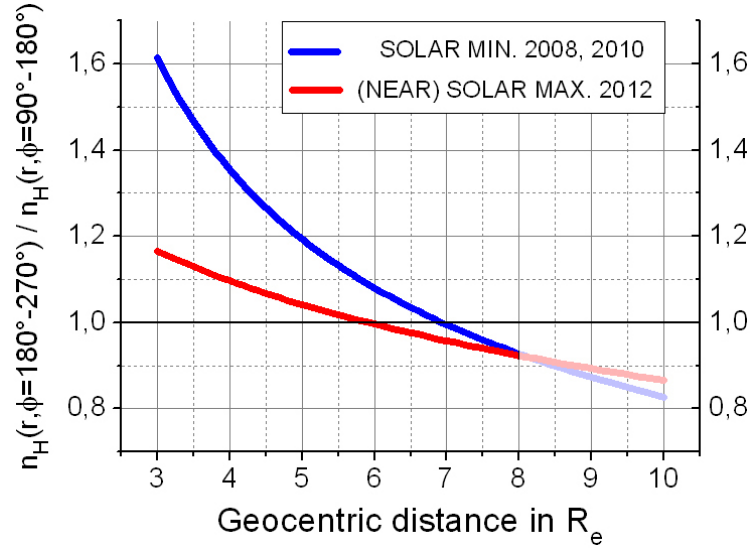

Figure 14. Dawn/dusk asymmetry plotted as the H-column density ratio for the regions between "midnight-dawn" and "midnightdusk" for the solar minimum (blue) and the solar maximum (red) model fit. Between 8 and $10 R_{\mathrm{e}}$ the plot shows model-extrapolated values, which might have a larger uncertainty compared to $8 R_{\mathrm{e}}$ (light colored).

visible in Fig. 14 for solar minimum at $\approx 7 R_{\mathrm{e}}$ and for solar maximum at $\approx 6 R_{\mathrm{e}}$ ).

\section{Polar density depletion}

The exospheric H-density profiles over the polar regions (GSE ecliptic north and south pole) are noticeably depleted compared to the (averaged) H-density profile within the ecliptic plane. In general, this depletion effect is visible independent of the solar activity but differs in its strength. For the Northern Hemisphere the depletion appears a little weaker compared to the Southern Hemisphere. This difference might be real or an observational effect caused by the much better northern observational coverage.

By averaging both (ecliptic northern and southern) polar density profiles and comparing these to the ecliptic dusk density profile, we derive values of 0.74 (solar minimum) and 0.82 (solar maximum) for the (averaged) polar/ecliptic density depletion. Between 3 and $8 R_{\mathrm{e}}$ these values have a small variation with distance.

\section{Conclusions}

This work presents model fits of the 3-D H-density distribution of the terrestrial exosphere for different solar activities. Our density inversion is based on exospheric TWINS stereo Lyman- $\alpha$ column brightness observations. The results contain density uncertainties of about 15-20\% and have the validity range between 3 and $8 R_{\mathrm{e}}$ geocentric distances.

We have noticed higher densities and a larger extension into circumterrestrial space of the neutral exosphere under 
(near) solar maximum conditions (2012) compared to solar minimum conditions $(2008,2010)$.

Variations in the solar activity on a monthly timescale cause an observable change in the exospheric H-density profile. This is shown in two separate $N(r)$ fits for 20-22 October and 20-24 December 2012, with higher solar activity and density in 20-22 October as expected. We conclude that the systematic decrease in the averaged 27-day (total) solar Lyman- $\alpha$ flux within these 2 months is accompanied by a cooling process that reduces density, temperature and (spatial) extension of the $H$ exosphere in a measurable manner.

The TWINS Lyman- $\alpha$ observation are corrected for geocoronal multiple-scattering effects (i.e., geocoronal selfabsorption or re-emission), which increases the number of useable LOSs (particularly on the Earth's nightside) within the model fit. The local correction factors $\epsilon(r, \theta, \phi)$ for geocoronal self-absorption and re-emission were calculated based on a radiation transport MCM using "coherent linecentered" multiple scattering of Lyman- $\alpha$ photons as an approximation.

At the Earth's nightside, a region with significantly enhanced $\mathrm{H}$ densities appears between midnight and dawn. From $3 R_{\mathrm{e}}$ with increasing distance $r$ this region is shifted back to midnight and beyond. This "H-geotail" phenomenon has some structural differences for different solar activities. Nevertheless, for solar minimum as well as for solar maximum, the $\mathrm{H}$ geotail at $8 R_{\mathrm{e}}$ has its density peak opposite to the incoming solar wind, which is aberrated about $-4^{\circ}$ to the Earth-Sun line.

The dawn-dusk asymmetry during solar minimum (see Zoennchen et al., 2013) was confirmed and found to be smaller for solar maximum. In this analysis it turned out that this asymmetry with higher dawn densities could be a side effect of the $\mathrm{H}$ geotail, which is significantly dawnshifted at lower distances. The dawn-dusk asymmetry seems to be inverted (with higher dusk densities) at larger distances (6-7 $R_{\mathrm{e}}$ ), where the $\mathrm{H}$ geotail is close to being solar-windaligned and no longer dawn-shifted.

The longitudinal orientation of the exosphere at lower distances $\left(3 R_{\mathrm{e}}\right)$ differs from the orientation at larger distances $\left(8 R_{\mathrm{e}}\right)$. A possible explanation might be that different interaction processes are forming and orientating the lower and the outer exosphere. Because of its orientation along the Earthsolar-wind line at $8 R_{\mathrm{e}}$, the outer exosphere seems to be influenced by interactions with solar wind ions or ions from the outer magnetosphere. The lower exosphere at 3-4 $R_{\mathrm{e}}$ seems to be dominated by orbiting $\mathrm{H}$ atoms which scattered into orbits post-noon (13:00-14:00 LT) on the Earth's dayside and form a region of enhanced density near their apogees between midnight and dawn.
Acknowledgements. The authors gratefully thank the TWINS team (PI Dave McComas) for making this work possible. We also acknowledge the support by the German Federal Ministry of Economics and Technology (BMWi) through the DLR grants FKZ 50 OE 0901 and FKZ 50 OE 1401. Additionally, we thank both of the referees, especially Jerry Goldstein, for the discussions and the very extensive help with improving the paper.

Topical Editor A. J. Kavanagh thanks J. Goldstein and one anonymous referee for their help in evaluating this paper.

\section{References}

Anderson, D. E. and Hord Jr., C. W.: Multidimensional radiative transfer - Applications to planetary coronae, Planet. Space Sci., 25, 563-571, doi:10.1016/0032-0633(77)90063-0, 1977.

Bailey, J. and Gruntman, M.: Experimental study of exospheric hydrogen atom distributions by Lyman- $\alpha$ detectors on the TWINS mission, J. Geophys. Res., 116, A09302, doi:10.1029/2011JA016531, 2011.

Bailey, J. and Gruntman, M.: Observations of exosphere variations during geomagnetic storms, Geophys. Res. Lett., 40, 1907-1911, doi:10.1002/grl.50443, 2013.

Bertaux, J. L., Pellinen, R., Chassefiere, E., Dimarellis, E., Goutail, F., Holzer, T. E., Kelha, V., Korpela, S., Kyrölä, E., and Lallement, R.: SWAN: A study of solar wind anisotropies, In ESA, The SOHO Mission, Scientific and Technical Aspects of the Instruments, 63-68 (SEE N90-13302 04-92), 1998.

Bishop, J.: Analytic exosphere models for geocoronal application, Planet. Space Sci., 39, 885-893, 1991.

Brandt, J. C. and Chamberlain, J. W.: Hydrogen Radiation in the Night Sky, Astrophys. J., 130, 670-682, doi:10.1086/146756, 1959.

Brasken, M. and Kyrölä, E.: Resonance scattering of Lyman alpha from interstellar hydrogen, Astron. Astrophys., 332, 732-738, 1998.

Bzowski, M.: A model of Charge Exchange of Interstellar Hydrogen on a Time-Dependent 2D Solar Wind, Space Sci. Rev., 97, 379-383, 2001.

Bzowski, M., Möbius, E., Tarnopolski, S., Izmodenov, V., and Gloeckler, G.: Density of neutral interstellar hydrogen at the termination shock from Ulysses pickup ion observations, Astron. Astrophys., 491, 7-19, 2008.

Bzowski, M., Sokól, J. M., Tokumaru, M., Fujiki, K., Quémerais, E., Lallement, R., Ferron, S., Bochsler, P., and McComas, D. J.: Cross-Calibration of Far UV Spectra of Solar System Objects and the Heliosphere, ISSI Scientific Report Series, Volume 13. ISBN 978-1-4614-6383-2, Springer Science \& Business Media New York, 67-138, doi:10.1007/978-1-4614-6384-9_3, 2013.

Carruthers, G. R., Page, T., and Meier, R. R.: Apollo 16 Lyman alpha imagery of the hydrogen geocorona, J. Geophys. Res., 81, 1664-1672, 1976.

Chamberlain, J. W.: Planetary coronae and atmospheric evaporation, Planet, Space Sci., 11, 901-960, 1963.

Costa, J., Lallement, R., Quémerais, E., Bertaux, J. L., Kyrölä, E., and Schmidt, W.: Heliospheric interstellar $\mathrm{H}$ temperature from SOHO/SWAN H cell data, Astron. Astrophys., 349, 660-672, 1999. 
Emerich, C., Lemaire, P., Vial, J.-C., Curdt, W., Schühle, U., and Wilhelm, K.: A new relation between the central spectral solar HI Lyman- $\alpha$ irradiance and the line irradiance measured by SUMER/SOHO during the cycle 23, Icarus, 178, 429-433, doi:10.1016/j.icarus.2005.05.002, 2005.

Fahr, H. J.: The Interplanetary Hydrogen Cone and its Solar Cycle Variations, Astron. Astrophys., 14, 263-274, 1971.

Fahr, H. J. and Shizgal, B.: Modern Exospheric Theories and Their Observational Relevance, Rev. Geophys. Space Phys., 21, 75124, 1983.

Hodges Jr., R. R.: Monte Carlo simulation of the terrestrial hydrogen exosphere, J. Geophys. Res., 99, 23229-23247, 1994.

Johnson, F. S.: The Distribution of Hydrogen in the Telluric Hydrogen Corona, Astrophys. J., 133, 701-705, doi:10.1086/147072, 1961.

Kupperian, J. E., Byram, E. T., Chubb, T. A., and Friedman, H.: Far ultra-violet radiation in the night sky, Planet. Space Sci., 1, 3-6, doi:10.1016/0032-0633(59)90015-7, 1959.

Kyrölä, E., Summanen, T., Schmidt, W., and Mäkinen, T.: Preliminary retrieval of solar wind latitude distribution from Solar Wind Anisotropies/SOHO observations, J. Geophys. Res., 103, 1452314538, 1998.

McComas, D. J., Allegrini, F., Baldonado, J., Blake, B., Brandt, P. C., Burch, J., Clemmons, J., Crain, W., Delapp, D., Demajistre, R., Everett, D., Fahr, H., Friesen, L., Funsten, H., Goldstein, J., Gruntman, M., Harbaugh, R., Harper, R., Henkel, H., Holmlund, C., Lay, G., Mabry, D., Mitchell, D., Nass, U., Pollock, C., Pope, S., Reno, M., Ritzau, S., Roelof, E., Scime, E., Sivjee, M., Skoug, R., Sotirelis, T. S., Thomsen, M., Urdiales, C., Valek, P., Viherkanto, K., Weidner, S., Ylikorpi, T., Young, M., and Zoennchen, J.: The Two Wide-angle Imaging Neutral-atom Spectrometers (TWINS) NASA Mission-of-Opportunity, Space Sci. Rev., 142, 157-231, doi:10.1007/s11214-008-9467-4, 2009.

McComas, D. J., Angold, N., Elliott, H. A., Livadiotis, G., Schwadron, N. A., Skoug, R. M., and Smith, C. W.: Weakest Solar Wind of the Space Age and the Current "Mini" Solar Maximum, Astrophys. J., 779, 10 pp., doi:10.1088/0004637X/779/1/2, 2013.

Nass, H. U., Zoennchen, J. H., Lay, G., and Fahr, H. J.: The TWINSLAD mission: Observations of terrestrial Lyman- $\alpha$ fluxes, Astrophys. Space Sci. Trans., 2, 27-31, doi:10.5194/astra-2-27-2006, 2006.
Østgaard, N., Mende, S. B., Frey, H. U., Gladstone, G. R., and Lauche, H.: Neutral hydrogen density profiles derived from geocoronal imaging, J. Geophys. Res. Space Phys., 108(A7), SMP 18, 1-12, doi:10.1029/2002JA009749, 2003.

Rairden, R. L., Frank, L. A., and Craven, J. D.: Geocoronal imaging with Dynamics Explorer, J. Geophys. Res., 91, 13613-13630, 1986.

Snow, M., Reberac, A., Quémerais, E., Clarke, J., McClintock, W. E., and Woods, T. N.: A New Catalog of Ultraviolet Stellar Spectra for Calibration, Cross-Calibration of Far UV Spectra of Solar System Objects and the Heliosphere, ISSI Scientific Report Series, Volume 13, ISBN 978-1-4614-6383-2, Springer Science \& Business Media New York, 191-226, 2013.

Sokól, J. M., Bzowski, M., Tokumaru, M., Fujiki, K., and McComas, D. J.: Heliolatitude and Time Variations of Solar Wind Structure from in situ Measurements and Interplanetary Scintillation Observations, Solar Phys., 285, 167-200, doi:10.1007/s11207-012-9993-9, 2013.

Thomas, G. E.: The interstellar wind and its influence on the interplanetary environment, Annual review of earth and planetary sciences, 6, (A78-38764 16-42) Palo Alto, Calif., Annual Reviews, Inc., 1978, 173-204, 1978.

Thomas, G. E. and Bohlin, R. C.: Lyman-alpha measurements of neutral hydrogen in the outer geocorona and in interplanetary space, J. Geophys. Res., 77, 2752-2761, 1972.

Witt, N., Blum, P. W., and Ajello, J. M.: Solar wind latitudinal variations deduced from Mariner 10 interplanetary H (1216 ̊) observations, Astron. Astrophys., 73, 272-281, 1979.

Woods, T. N., Tobiska, W. K., Rottman, G. J., and Worden, J. R.: Improved solar Lyman alpha irradiance modeling from 1947 through 1999 based on UARS observations, J. Geophys. Res., 105, 27195-27215, doi:10.1029/2000JA000051, 2000.

Zoennchen, J. H., Bailey, J. J., Nass, U., Gruntman, M., Fahr, H. J., and Goldstein, J.: The TWINS exospheric neutral H-density distribution under solar minimum conditions, Ann. Geophys., 29, 2211-2217, doi:10.5194/angeo-29-2211-2011, 2011.

Zoennchen, J. H., Nass, U., and Fahr, H. J.: Exospheric hydrogen density distributions for equinox and summer solstice observed with TWINS1/2 during solar minimum, Ann. Geophys., 31, 513527, doi:10.5194/angeo-31-513-2013, 2013. 